\title{
Reacciones psicológicas ante el diagnóstico de COVID-19: un estudio cualitativo
}

Miguel Landa-Blanco ${ }^{1}$, Elizabeth Andino-Rodríguez ${ }^{2}$, Gabriela Vásquez ${ }^{3}$ y Miguel Ángel RuchOrdóñez ${ }^{4}$

Escuela de Ciencias Psicológicas de la Universidad Nacional Autónoma de Honduras (UNAH)

Nota de los autores:

${ }^{1}$ Coordinador de Investigación y Vinculación de la Maestría en Psicología Clínica de la UNAH. ORCID: 0000-0002-7865-7593. Correo electrónico: miguel.landa@unah.edu.hn

${ }^{2}$ Asistente de Investigación de la Maestría en Psicología Clínica de la UNAH. ORCID: 00000001-6969-1485

${ }^{3}$ Investigadora de la Maestría en Psicología Clínica de la UNAH. ORCID: 0000-0002-6982-8362

${ }^{4}$ Investigador en formación, Escuela de Ciencias Psicológicas de la UNAH. ORCID: 0000-00019279-0748 


\section{Resumen}

El presente estudio tuvo como objetivo conocer las experiencias y reacciones psicológicas asociadas al diagnóstico de COVID-19 en población hondureña. La metodología utilizada se enmarca en un enfoque cualitativo, con un diseño narrativo. La información fue recolectada por medio de entrevistas realizadas a un total de 35 sujetos adultos que hubiesen sido diagnosticados con COVID-19. Adicionalmente, como punto de triangulación, se incluyeron 5 entrevistas a personal médico trabajando en primera línea contra el COVID-19. Entre los temas prevalentes en el discurso de los informantes se evidencia que el diagnóstico positivo de COVID-19 tuvo efectos en el desarrollo cotidiano del sujeto, específicamente en lo referente a aspectos laborales, económicos y académicos. Además, los informantes comentan una serie de reacciones psicológicas, incluyendo sentimientos de culpa, ansiedad, depresión, preocupación por la salud propia y de los seres queridos, deterioro en la calidad de sueño, incertidumbre, temor y distintas actitudes hacia la búsqueda de atención psicológica. También se menciona la importancia de las redes de apoyo, puntualmente en lo referente a la familia, amistades y compañeros de trabajo. En ocasiones los entrevistados reportaron sentimientos de soledad y ser víctimas de estigmatización. Estos resultados se discuten a la luz de los antecedentes y sus implicaciones prácticas.

Palabras clave: Psicología de la salud; pandemia; COVID-19; salud mental; bienestar psicológico 


\title{
Psychological reactions to the positive diagnosis of COVID-19: a qualitative study
}

\begin{abstract}
The present study aimed to understand the experiences and psychological reactions associated with the diagnosis of COVID-19 in the Honduran population. The methodology used was framed within a qualitative approach, with a narrative design. The information was collected through interviews with a total of 35 adult subjects who had been diagnosed with COVID-19. Additionally, as a triangulation point, 5 interviews with medical personnel working on the front line against COVID19 were included. Among the prevailing matters in the informants' discourse, it is evident that the positive diagnosis of COVID-19 had effects on the daily development of the individual, specifically in relation to labor, economic and academic aspects. In addition, the informants comment on a series of psychological reactions, including feelings of guilt, anxiety, depression, concern for their own health and that of loved ones, deterioration in the quality of sleep, uncertainty, fear, and different attitudes towards seeking psychological help. The importance of support networks is also mentioned, specifically in relation to family, friends, and co-workers. Sometimes the interviewees reported feelings of loneliness and being victims of stigmatization. These results are discussed in light of previous research and their practical implications.
\end{abstract}

Keywords:Health psychology; pandemic; COVID-19; mental health; psychological wellbeing 


\section{Introducción}

\section{Origen y prevalencia del COVID-19}

A finales del año 2019 se identificó en Wuhan, China, un nuevo tipo de virus llamado SARS-CoV-2 y su enfermedad asociada como COVID-19 (Shereen, Khan, Kazmi, Bashir, \& Siddique, 2020). Para el 11 de marzo de 2020, la Organización Mundial de la Salud (OMS) etiquetó la situación de COVID-19 como pandemia (OMS, 2020). La infección del COVID-19 puede causar síntomas que varían de leves a graves, y en algunos casos puede ser mortal. Los síntomas pueden incluir: fiebre, tos, dolor de garganta, dolor de cabeza, secreción nasal, neumonía, entre otros (Hui \& Zumla, 2019). Cierto número de personas infectadas por el virus manifestaron haber experimentado otros síntomas no respiratorios, mientras otras personas fueron referidas como casos asintomáticos y no informaron haber experimentado ningún síntoma (Secretaria de Salud de Honduras, 2020). En cuanto a la prevención, mantener el distanciamiento social y el uso de la mascarilla han sido las medidas más eficientes para reducir el suceso de la propagación (Centros para el Control y la Prevención de Enfermedades [CDC], 2020).

Para finales del año 2020, a nivel mundial se reportaban 79,231,893 casos confirmados de COVID-19 y 1,754,574 de muertes. En el continente americano se concentra el $43 \%$ de los casos acumulados de COVID-19 y $47 \%$ de las muertes. En Honduras los primeros casos de la enfermedad se confirman el 10 de marzo del año 2020 (Secretaria de Salud de Honduras, 2020b). Para finales del año 2020 el país reportaba 118,659 casos con 3,061 fallecimientos atribuidos al COVID-19 (OMS, 2020).

\section{Reacciones psicológicas ante el diagnóstico de COVID-19}

El diagnóstico de enfermedades potenciales mortales es un evento estresante para los pacientes (McBride, Clipp, Peterson, Lipkus, \& Demark-Wahnefried, 2000). La información 
acerca de la salud mental de los pacientes que han sido diagnosticados con COVID-19 se encuentra aún en desarrollo debido a que la pandemia sigue vigente y cada día se identifican nuevos hallazgos al respecto. Un estudio reciente encontró que al momento de ser diagnosticados con COVID-19, el $74 \%$ de la muestra reportó haber entrado en un estado de "shock", $60 \%$ reportó sentirse triste, 68\% en pánico o con ansiedad, y el 54\% se mostró incrédulo. Este mismo estudio identificó que al recibir el diagnóstico, uno de cada seis pacientes pensó con total certeza que moriría (Sahoo, y otros, 2020).

Además, existe evidencia científica de que hay diferencias significativas en cuanto a aspectos de salud mental entre diferentes poblaciones, por ejemplo, personas que solo han estado bajo cuarentena y aislamiento, personas con diferentes niveles de exposición al virus, y el resto de la población, encontrando que la vulnerabilidad psicológica está relacionada a factores como el género, edad, exposición a información mediática, estado civil, entre otros (Zhang, y otros, 2020). De igual forma, se ha encontrado que, específicamente relacionado a síntomas psicológicos, los niveles de ansiedad y depresión son más altos en pacientes de COVID-19, tanto en casos confirmados como sospechosos, que en los de la población en general (Paz, y otros, 2020). Además, se ha identificado un aumentado en reportes de manifestaciones neurológicas y neuropsiquiátricas en pacientes de COVID-19 que tienen comorbilidad con trastornos psicológicos y/o psiquiátricos como esquizofrenia (Zandifar \& Badrfam, 2020), aumento de casos de brotes psicóticos no reactivos y delirios, llevando a diagnóstico de trastorno psicótico debido a otra condición médica, en este caso, COVID-19 (Majadas, y otros, 2020); y, basado en epidemias pasadas, el estigma relacionado a la exposición de la enfermedad puede generar aumento en sintomatología psicosomática, lo cual es exacerbado por la desinformación y el temor a lo desconocido (Muhidin, Vizheh, \& Moghadam, 2020). 
Reacciones psicológicas ante el diagnóstico de COVID-19: un estudio cualitativo

Debido a lo anterior, algunos autores han sistematizado diferentes intervenciones para el cuidado de los pacientes y sus familiares con actividades como el desarrollo de terapias presenciales y virtuales, manejo de complicaciones psicológicas como ansiedad, estrés, depresión, apoyo a familias en cuarentena, procesos de duelo, entre otros (Urzúa, Vera-Villarroel, CaqueoUrízar, \& Polanco-Carrasco, 2020).

\section{Propósito del estudio}

El presente estudio tiene como propósito estudiar las experiencias y construcciones subjetivas de personas que han sido diagnosticados con COVID-19. De manera particular, se pretende explorar las dimensiones psicológicas, sociales y económicas asociadas a la vivencia del diagnóstico positivo de COVID-19 en población hondureña. Se espera que estos insumos sirvan para elaborar intervenciones de acompañamiento psicológico a lo largo del proceso de diagnóstico y desarrollo del COVID-19 en población adulta.

\section{Materiales y métodos}

\section{Participantes}

La selección no probabilística de informantes se realizó en el marco de los siguientes criterios de inclusión: 1) ser mayor de 18 años al momento de la entrevista, 2) haber tenido un diagnóstico positivo de COVID-19, 3) residir en Honduras, y 4) consentir participar en la investigación. Se realizaron un total de 35 entrevistas, de estas el $68 \%$ corresponden al sexo femenino y $32 \%$ al masculino. La edad promedio de los informantes fue de 30.06 años, con una desviación estándar de 11.31 años, oscilando entre los 19 y 61 años. El 20\% de los participantes reportaron haber sido asintomáticos, mientras que el restante $80 \%$ había tenido síntomas de 
Reacciones psicológicas ante el diagnóstico de COVID-19: un estudio cualitativo

COVID-19. Dada la recurrencia en el contenido de las entrevistas se argumenta el alcance de la saturación teórica. Adicionalmente, con el fin de aumentar la credibilidad de los resultados, se entrevistaron a cinco médicos hondureños en primera línea contra el COVID-19. La información de tales entrevistados servirá de punto de triangulación para los hallazgos.

\section{Diseño}

La investigación se enmarcó en un enfoque cualitativo, procurando una comprensión de la experiencia subjetiva de los participantes en lo referente al fenómeno de estudio (Hernández, Fernández, \& Baptista, 2014). De manera específica se utiliza un diseño narrativo de tipo descriptivo, lo que indica la preponderancia del discurso de los informantes como expresión de su experiencia subjetiva.

\section{Técnicas de recolección de información}

Se elaboró una guía de entrevista semiestructurada. La pauta básica constaba de un total de 18 preguntas que indagaban respecto a las experiencias y reacciones psicológicas de los participantes entorno al diagnóstico de COVID-19. Algunas de las preguntas incluidas son: “¿Cómo describiría su reacción al momento de recibir los resultados del diagnóstico?”, “En qué áreas de su vida siente que le afectó al ser diagnosticado por COVID-19?”, “AAl ser diagnosticado(a) con COVID-19, buscó o no algún tipo de ayuda psicológica? ¿Por qué?”, entre otras. Además, se indagaba respecto a datos generales del informante, como el sexo, edad, personas con quien habita, condición laboral y sintomatología. Las entrevistas fueron llevadas a cabo de manera virtual, guardando una grabación en audio de las sesiones.

\section{Procedimiento}


Una vez elaborada la pauta de entrevista y delimitados los criterios de inclusión, se realizó una identificación no-probabilísticas de personas que hubiesen tenido un diagnóstico positivo de COVID-19. A los potenciales participantes se les compartió el Consentimiento Informado y se procedió a agendar una sesión de entrevista por medios virtuales. Al inicio de cada entrevista se dio lectura del Consentimiento Informado y se confirmó la voluntad de participación de los sujetos. La entrevista fue grabada en audio y posteriormente transcrita para luego ser codificadas en temas y subtemas coherentes con el propósito del estudio. Además, se realizaron entrevista a personal médico laborando en primera línea contra el COVID-19. La información provista por tales profesionales permitió una triangulación de la información.

\section{Análisis de datos}

Las entrevistas fueron grabadas en audio y posteriormente transcritas para luego realizar un análisis temático de los hallazgos. Para ello los fragmentos textuales fueron clasificados en subcategorías, las cuales a la vez se agruparon en categorías temáticas (Hernández, Fernández, \& Baptista, 2014). Luego, se realizaron tres niveles de triangulación, la primer entre los mismos informantes, la segunda entre actores, particularmente personal médico en primera línea contra el COVID-19 y finalmente una triangulación contra teoría/antecedentes expuesta en la discusión del presente artículo.

\section{Consideraciones éticas}

Se solicitó el consentimiento informado de los participantes, asegurando los principios de voluntariedad y confidencialidad. Además, se siguieron los lineamientos éticos establecidos por el 
Reacciones psicológicas ante el diagnóstico de COVID-19: un estudio cualitativo

programa de la Maestría de Psicología Clínica de la Universidad Nacional Autónoma de Honduras (UNAH). Además, la investigación se apega a los lineamientos establecidos en la Declaración Universal de Principios Éticos para Psicólogos.

\section{Resultados}

A continuación, se presentan los principales resultados del estudio. Entre los temas prevalentes en el discurso de los informantes se evidencia que el diagnóstico positivo de COVID19 tuvo efectos en el desarrollo cotidiano del sujeto, específicamente en lo referente a aspectos laborales, económicos y académicos. Además, los informantes comentan una serie de reacciones psicológicas, incluyendo ansiedad, depresión, preocupación por la salud propia y de los seres queridos, deterioro en la calidad de sueño, incertidumbre, temor y distintas actitudes hacia la búsqueda de atención psicológica. También se menciona la importancia de las redes de apoyo, puntualmente en lo referente a la familia, amistades y compañeros de trabajo. En ocasiones los entrevistaron reportaron sentimientos de soledad y ser víctimas de estigmatización, ver Figura 1.

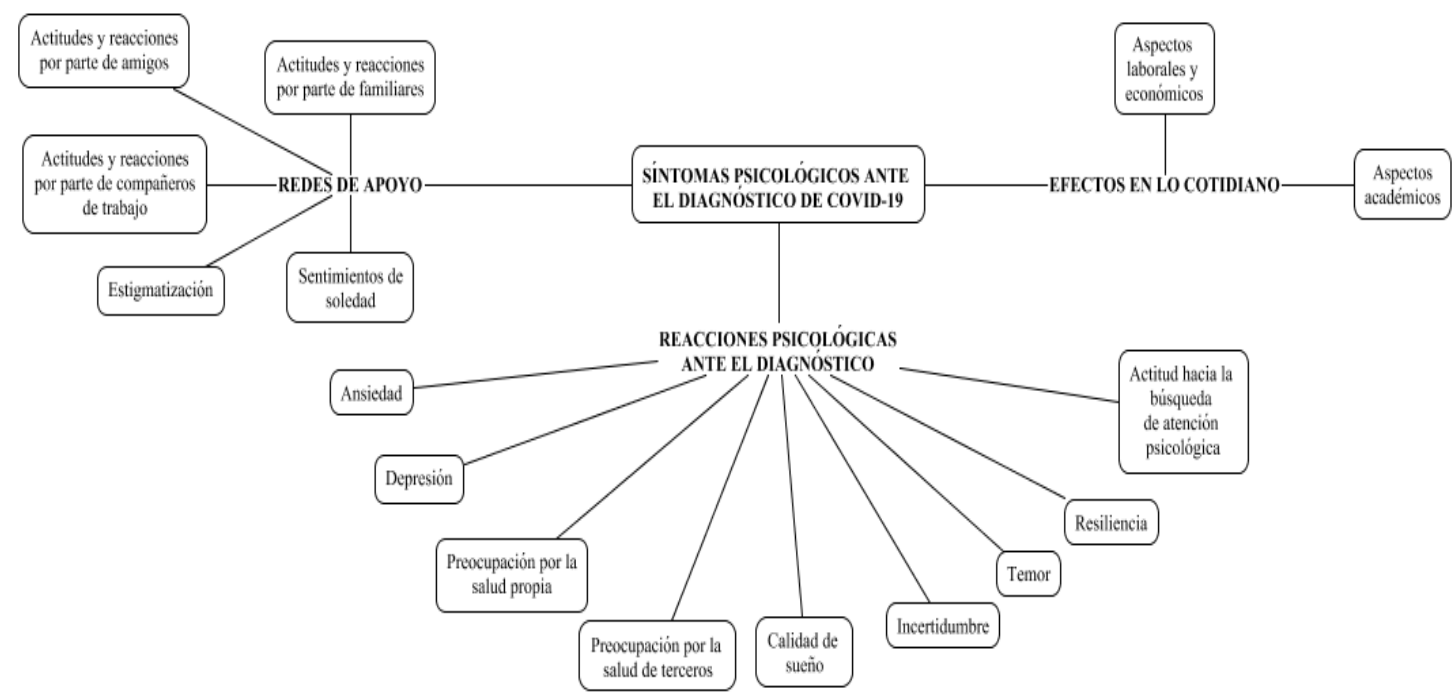

Figura 1: Diagrama de temas y subtemas presentes en el discurso de los informantes 


\section{Efectos en lo cotidiano}

Aspectos laborales y económicos

El diagnóstico de COVID-19 trae consigo diferentes consecuencias a nivel laboral dependiendo de la situación de cada uno de los pacientes. Para el caso, personas que trabajan en negocios familiares y de emprendimiento se ven obligados a detener las visitas a clientes lo que significa la pérdida momentánea de ingresos económicos. Por otra parte, personas que trabajan in situ se ven impactados en el sentido de cambio a nuevas áreas de desempeño donde deben permanecer aislados, lo que puede provocar sensación de rechazo, como se evidencia en los siguientes comentarios:

"Yo estaba trabajando en el área de ventas, yo tenía que andar visitando a los clientes, muchas de las empresas cerraron o nos cancelaron los contratos, no están haciendo implementación de nada, no están trabajando porque tienen parados los negocios, no están haciendo ningún tipo de negocio o inversión y eso ha venido a afectar gravemente mi economía porque ya no tenemos clientes que visitar, no tengo empresas a donde llevar los servicios. Estamos ahorita en una situación muy difícil”. Mujer, 37 años, presentó síntomas.

Además de los aspectos mencionados anteriormente, los entrevistados comentan acerca del impacto que tiene el diagnóstico en el área económico puesto que debían incurrir en nuevos gastos relacionados a las pruebas, tratamientos médicos, implementos de bioseguridad, entre otros, como se evidencia en el siguiente relato:

"Si, bastante, en el área económica porque se gastó bastante, o sea, mi familia y yo tuvimos que alquilar un tanque de oxígeno y no es algo barato, se gastó también en inyecciones que me estuve inyectando como 2 semanas seguidas, tratamientos porque cuando yo me enfermé no había 
Reacciones psicológicas ante el diagnóstico de COVID-19: un estudio cualitativo

tanto acceso a los tratamientos como hay ahora de COVID, tampoco fui a un hospital público por el miedo". -Mujer, 23 años, presentó síntomas.

Evidentemente, la incertidumbre y/o deterioro en el área económica y laboral tiene repercusiones sobre la salud mental de los entrevistados. Además, existe una preocupación por la situación laboral, a lo cual se le suman los costos asociados al tratamiento del COVID-19. Es posible triangular los testimonios de los informantes con lo expuesto por el personal médico entrevistado:

"El no trabajar, por lo menos la mayoría de los pacientes a los que se les ha dado el diagnóstico, cuando ya salen de la etapa negación. Lo único que piensan es 'que no voy a trabajar', ‘¿Y cómo voy a hacer con el trabajo?’. Entonces les preocupa más el trabajo que la salud [...]. Y también a veces es muy difícil decirles que guarden la cuarentena, porque a veces muchos trabajan fuera. No sólo les afecta en el área social, sino también en el área del trabajo, verdad, porque muchos temen perder sus trabajos al salir positivos. "- Entrevista PM6, personal médico.

\section{Aspectos académicos}

Los aspectos académicos representan un área de gran importancia para las personas y de igual forma son un estresor significativo para los pacientes diagnosticados con COVID-19 ya que este es un impedimento para continuar con sus actividades diarias y por consiguiente podría ser razón de abandono de clases o la reprobación de estas. Al respecto los entrevistados comentan: 
Reacciones psicológicas ante el diagnóstico de COVID-19: un estudio cualitativo

"El hecho de las clases me preocupaba bastante no poder terminar el periodo con éxito y abandonar las clases porque ya estaba bastante avanzada, esas eran mis máximas preocupaciones en ese momento". - Mujer, 20 años, presentó síntomas.

"Yo también estudio la carrera de pedagogía a distancia y obviamente pues cuando estuve incapacitado no, no pude recibir clases, no pude cumplir con mis asignaciones y de hecho todavía estoy tratando de ponerme al día”. - Hombre, 47 años, presentó síntomas.

\section{Reacciones psicológicas ante el diagnóstico de COVID-19}

Ansiedad

La ansiedad es una reacción típica reportada por los entrevistados al momento de ser diagnosticados de COVID-19. Esta ansiedad está vinculada con la incertidumbre respecto a la salud, propia y de terceros, pero también respecto al aislamiento. Ante esto se comenta:

"Pues me sentí como ansiosa, porque estar encerrada implicaba estar sola en mi cuarto, entonces me sentía ansiosa, malhumorada, desanimada, eso me sentía." - Mujer, 22 años, presentó síntomas.

"Me sentí con miedo y ansiedad al no saber qué más poder hacer. Al darme el diagnóstico sólo me dijeron que tenía que estar en cuarentena y tomar acetaminofén y el miedo de no saber qué podía hacer para mejorar y el sentirme mejor cada día." - Hombre, 20 años, presentó síntomas.

Las narraciones anteriores pueden ser trianguladas con lo que comenta el personal médico entrevistado, quienes concuerdan que el diagnóstico de COVID-19 detona síntomas de ansiedad en los pacientes. En ocasiones incluso se detonan ataques de pánico. Según narran: 
Reacciones psicológicas ante el diagnóstico de COVID-19: un estudio cualitativo

"Pues los pacientes reaccionan en su mayoría muy ansiosos. Hemos tenido varios ataques de pánico, incluso en el triaje, por ser diagnosticados con COVID. ”- Entrevista PM5, personal médico.

\section{Depresión}

Otra de las reacciones que se repite en las narraciones de los participantes es la depresión por lo que implica sobrellevar la enfermedad. La mayor parte de los participantes sintieron tristeza al no poder tener contacto con las demás personas y al momento de estar solo en la habitación. Además, los entrevistados manifiestan que lloraban al sentirse solos y abrumados al haber contraído la enfermedad en una temporalidad donde existían muchos casos de muertes por COVID-19. Se evidencia con los siguientes fragmentos:

"Yo entre en depresión, porque más que todo porque estaba encerrada, aislada, en un cuarto de aquí de mi casa, y al escuchar a los niños que me llamaban y todo eso, porque están pequeños, entonces yo me sentí, así como que no podía atenderlos. Me sentía mal porque ellos necesitaban que su mamá estuviera ahí, y si sentía mucha tristeza que ellos querían estar conmigo y ellos no podían." - Mujer, 35 años, presentó síntomas.

Lo anterior concuerda con las entrevistas realizadas a personal médico, quienes indican que los síntomas depresivos son una reacción común en los pacientes diagnosticados. Cabe notar que el personal médico percibe que la intensidad de los síntomas depresivos varía según la gravedad del diagnóstico de COVID-19. Según narran:

"Los pacientes se ponían a llorar, reaccionaban de una forma ansiosa. Les preocupaba el bienestar de su familia. Se ponían tristes, incluso en los primeros meses donde estuvimos diagnosticando COVID-19, teníamos que usar ansiolíticos, antidepresivos también en algunos 
Reacciones psicológicas ante el diagnóstico de COVID-19: un estudio cualitativo

pacientes. Y claro está que la reacción es peor en los pacientes a mayor gravedad de la enfermedad."- Entrevista PM5, personal médico.

Preocupación por la salud propia

En el discurso de los informantes se evidencia una preocupación por las consecuencias del COVID-19 sobre la salud propia. Tal temor no se centraba únicamente en las complicaciones o síntomas asociados al virus, sino también al proceso de hospitalización. Cabe agregar que estas preocupaciones están asociadas a síntomas de angustia. Esto se evidencia en los siguientes testimonios:

"Mi mayor preocupación era que me complicara y me tuvieran que llevar al hospital de emergencia y me dejaran interna." - Mujer, 37 años, presentó síntomas.

El temor de los pacientes diagnosticados con COVID-19 a las secuelas de la enfermedad y a la muerte también ha sido percibido por el personal médico, quienes comentan:

"Piensa también que va a quedar con secuelas, algunos si quedan con secuelas, y entonces eso lo lleva a ese tipo de problemas. Puede que diga 'no voy a volver a ser la misma persona' [...]. La persona entra en esa desesperación, creo que nadie se quiere morir. Entonces entran en esa fase de desesperación. Y eso lo lleva a otro tipo de problemas, así de salud mental."- Entrevista PM2, personal médico.

Preocupación por la salud de terceros

Además de la preocupación por la propia salud los entrevistados narran su temor por la salud de los seres cercanos. Esta preocupación está particularmente dirigida a la familia, en 
Reacciones psicológicas ante el diagnóstico de COVID-19: un estudio cualitativo

especial a las poblaciones vulnerables, como aquellas personas de la tercera edad. Según comentan los entrevistados:

"Más que toda la preocupación mayor era contagiar a mi familia, sobre todo a mi mamá, que es la que está más cercana a mí."- Mujer, 20 años, presentó síntomas.

"Sentí que se me caía el mundo porque días atrás estuve con mi papá y él es diabético, sentí que se me venía el mundo abajo no pensé tanto en mí, sino que en mi papá y en si lo pude contagiar, fue una experiencia bien fea." - Mujer, 24 años con síntomas

"Por mi causa se contagió mi mamá, mi hermano, que gracias a Dios fue asintomático, pero mi mamá si presentó síntomas. Entonces si me afectó, como que me sentí un poco culpable en el momento de haber contagiado a mi mamá." -Mujer, 23 años, presentó síntomas.

Estos testimonios son triangulados con lo expuesto por el personal médico, quienes comentan:

"Se preocupaban por sus familiares y por todas las personas con las que habían tenido contacto [...] y si es un paciente joven, preocuparse por la muerte de sus familiares o de su mamá o papá, que son las personas más afectadas. ”- Entrevista PM3, personal médico.

\section{Calidad de sueño}

Los entrevistados testifican de un deterioro en la calidad de su sueño. Pero existen situaciones diferenciadas en donde algunos reportan hipersomnia, mientras que otros experimentaron insomnio. Esto se evidencia en los siguientes fragmentos de entrevista:

“El dolor de cabeza no me dejaba dormir.”- Hombre, 20 años, presentó síntomas. 
Reacciones psicológicas ante el diagnóstico de COVID-19: un estudio cualitativo

"La verdad mala, porque me sentía temeroso de igual manera era bien interrumpido la verdad, no fue placentero al estar con eso me afectó la fiebre y no tenía un sueño constante y profundo."- Hombre, 31 años, presentó síntomas.

"Dormía mucho, tanto como en el día como en la noche, el agotamiento era extremo, me sentía bastante cansada, había días que solo quería dormir, dormir y dormir."- Mujer, 35 años, presentó síntomas.

\section{Incertidumbre}

Una de las emociones más recurrentes en el discurso de los entrevistados es la incertidumbre. En este sentido, durante el padecimiento de la enfermedad, algunas personas expresaron una inquietud sobre el futuro, estados de salud y la posibilidad de fallecer. Además, la inseguridad que existía con respecto al contagio a otros o las repercusiones que podrían aparecer luego. Sobre esto los entrevistados comentan:

“Creo que lo más difícil fue el hecho de que, como mi diagnóstico era bastante negativo no sabíamos en realidad qué era lo que iba a pasar conmigo, entonces creo que lo más difícil para mí fue saber que no sabía que iba a pasar con mi vida, o sea llegué al punto que yo me sentía tan mal que yo me dije a mi misma en mi mente "no sé si me voy a morir" por qué me sentía bien mal." - Mujer, 24 años, presentó síntomas.

Temor

Otro factor relevante es el temor hacía el COVID-19 y sus consecuencias. Muchos comentaban sobre el momento de recibir el diagnóstico los sentimientos que los abrumaba, como 
Reacciones psicológicas ante el diagnóstico de COVID-19: un estudio cualitativo

miedo y angustia ante la posibilidad de muerte. Dos de los informantes expresan lo siguiente al respecto:

"Fue sorprendente y me dio temor, por las consecuencias y temor a morir, porque es un virus muy potente con riesgo de muerte y secuelas muy graves (...) temor a no saber cómo va a reaccionar el cuerpo a medida van cambiando los síntomas." -Mujer, 34 años, presentó síntomas.

"Sentí miedo, fue difícil y entró el temor a mi vida, tuve miedo a perder la vida por esa enfermedad." -Hombre, 49 años, presentó síntomas.

\section{Resiliencia}

Unas de las reacciones manifestadas que se repiten en el discurso de los participantes es la resiliencia que tuvieron en los momentos de ser diagnosticados y sobrellevando la enfermedad de COVID-19. La mayor parte de los entrevistados se cimentaba en su fe religiosa y convencidos por sus experiencias religiosas que iban a lograr superarlo, una de las entrevistas comenta:

"Yo soy una persona muy religiosa y esta experiencia me sirvió mucho para acércame más a Dios porque llegó un punto en el que solo éramos él y yo." -Mujer, 24 años, presentó síntomas.

De la misma forma, manifestaban realizar algunas actividades como ver videos motivacionales, mantener un pensamiento positivo, estar activo, escribir sus experiencias e incluso buscar consuelo con personas de su círculo cercano. Por lo tanto, analizando el discurso de cada uno de ellos, se evidencia que su manera de sobreponerse a esta enfermedad letal fue por medio de la búsqueda de fuentes de fortaleza y esperanza, expresando lo siguiente:

"Encomendándome a Dios, mis oraciones y las de mi familia eso me ayudó bastante y así fue como pude salir adelante... Miraba videos motivacionales para no caer en depresión, eso me 
Reacciones psicológicas ante el diagnóstico de COVID-19: un estudio cualitativo

ayudó bastante, también escribía todo lo que me pasaba, de hecho, tengo un par de videos en mis redes que gracias a ese proceso pude salir adelante." - Mujer, 20 años, presentó síntomas.

Actitud hacia la búsqueda de atención psicológica

La comunicación de un diagnóstico puede ser impactante para una persona, más complejo cuando se trata de una enfermedad desconocida y con una tasa de letalidad alta, que puede llevar a la persona a una desorganización mental. A pesar de esto, algunos entrevistados confesaron que no existe accesibilidad ni oportunidades de obtener una atención psicológica en los centros de salud. Además, los informantes manifestaron estar dispuestos a recibir ayuda psicológica, aunque no les dio tiempo de contemplarlo. Sin embargo, algunos informantes consideran que hubiera sido beneficioso:

“No busqué ayuda psicológica. No sé, no lo pensé en ese momento, pero quizás hubiera sido favorable haber buscado algún tipo de ayuda.”- Mujer, 25 años, presentó síntomas.

"No busqué porque en aquel momento no teníamos tantas oportunidades de consultas, de medios, solamente tuvimos acceso a consulta médica, no psicológica, creo que no por algún estigma, sino porque simplemente en aquel momento no era tan fácil el acceso a estos servicios de salud." - Hombre, 22 años, presentó síntomas.

Otra parte de los participantes manifestaron una actitud renuente a la atención psicológica, ya que creían que era algo innecesario o no lo percibían como una prioridad. Dos entrevistados comentan lo siguiente en este aspecto:

“No, no busqué. Realmente no, no lo vi necesario. No lo contemplé en ningún momento la verdad."- Mujer, 37 años, presentó síntomas 
Reacciones psicológicas ante el diagnóstico de COVID-19: un estudio cualitativo

"Realmente no, no busqué, y no busque porque no sé, no sé, cómo yo conozco de la psicología, considero yo, en mi mente que no debería buscar, pero si debería de buscar, pero honestamente no lo miro como una prioridad en este momento porque yo he aprendido a controlar eso, entonces no lo miro como una prioridad."-Mujer, 24 años, presentó síntomas

Los testimonios de los pacientes son triangulados con lo expuesto por el personal médico entrevistado, quienes comentan que en la actualidad no se da un acompañamiento psicológico a los pacientes. A pesar de que perciben que la atención psicológica es relevante y necesaria en la actual pandemia, consideran que tanto pacientes como autoridades en el área de salud, no dan la importancia debida a la salud mental de los pacientes. A tal situación se suman los estigmas que hay entorno a la búsqueda de atención psicológica. Según comentan:

“Creo que las personas que dirigen esta pandemia no están comprendiendo el impacto emocional, el impacto psicológico que tienen los pacientes, entonces descuidan esa parte."Entrevista PM3, personal médico

"Las personas piensan que solamente porque se va al psicólogo o al psiquiatra, que ya está loco [...]. Es por la poca importancia que le dan a la salud mental, siento yo."- Entrevista PM1, personal médico

\section{Redes de apoyo}

Actitudes y reacciones por parte de familiares

En el discurso de los informantes surge el tema de las reacciones de los familiares al darse cuenta de que su pariente se encuentra con diagnóstico de COVID-19 y, tanto para pacientes que presentaron síntomas como para los asintomáticos las principales reacciones son de preocupación y angustia tanto por la enfermedad como por las enfermedades de base que pueden agravar el 
Reacciones psicológicas ante el diagnóstico de COVID-19: un estudio cualitativo

diagnostico, y sus actitudes son de apoyo y solidaridad con su familiar. Esto puede verse en los siguientes comentarios:

"Se preocuparon bastante porque soy asmático no sabía si iba a presentar síntomas y más que había un montón de gente contagiada que estaba muriendo, entonces estaba pasándola mal”. -Hombre, 19 años, no presentó síntomas.

"Se pusieron bastante preocupados, súper preocupados y ellos trataron de hacer lo mejor posible para que yo saliera rápido de eso”. -Mujer, 23 años, presentó síntomas.

Actitudes y reacciones por parte de amigos

Otro grupo que representa una fuente de apoyo para los entrevistados es el caso de los amigos o personas cercanas ya que, al igual que los familiares, tienden a mostrarse preocupados por la enfermedad, pero también se muestran solidarios y buscan dar ánimos por medio de palabras de aliento, oraciones y demás, como lo mencionan los siguientes entrevistados:

"Mis amigos y demás familiares estuvieron siempre apoyándonos y nos escribian y pasaban pendientes de cómo nos sentíamos, nos preguntaban no solo si teníamos síntomas sino cómo estábamos llevando el encierro y cómo nos sentíamos emocionalmente”. -Hombre, 20 años, presentó síntomas.

Actitudes y reacciones por parte de compañeros de trabajo

De igual forma, los compañeros de trabajo representan una parte importante dentro de las redes de apoyo ya que estos se muestran solidarios, dando palabras de ánimo y se mantienen al pendiente de la evolución de los entrevistados, aunque también se muestran preocupados por su propia salud ante una posible exposición al virus. Esto se evidencia en los siguientes fragmentos: 
Reacciones psicológicas ante el diagnóstico de COVID-19: un estudio cualitativo

“En la laboral no, más bien todos apoyándome, escribiéndome seguido, dándome palabras de aliento”. -Mujer, 31 años, presentó síntomas.

"Sorprendidos también y preocupados. La verdad es que tuve bastante apoyo de conocidos, amigos, compañeros de trabajo, mis jefes también me brindaron bastante apoyo”. Mujer, 23 años, presentó síntomas.

\section{Estigmatización}

En las narraciones de los entrevistados se identifica que algunos de ellos se sintieron estigmatizados por las demás personas. Esto se evidencia en los siguientes comentarios:

"Hay personas que lo estigmatizan. Se alejan, no preguntan ni cómo estás, simplemente te miran y te miran con temor por miedo a contagiarse o te dicen, no mire, mejor en otro momento."Mujer, 22 años, presentó síntomas

"Algunas personas lo discriminan cuando estas se enteran de que uno está contagiado."Hombre, 20 años, presentó síntomas

"Se siente horrible porque las personas te rechazan y te hacen a un lado."- Mujer, 24 años, no presentó síntomas

Sentimientos de soledad

Debido a las medidas de cuarentena requeridas a las personas con diagnóstico positivo de COVID-19, muchas veces estos pacientes experimentan sentimientos de soledad. En tales casos se reporta un anhelo por interactuar socialmente con otros, teniendo cercanía o contacto físico. A continuación, se presentan fragmentos del discurso de los entrevistados en donde se evidencia tal situación: 
Reacciones psicológicas ante el diagnóstico de COVID-19: un estudio cualitativo

"Me afectó psicológicamente porque me ponía a llorar y me preguntaba ¿¿cuándo se me va a quitar esta enfermedad?', ya que yo los miraba todos juntos y yo aislada ya que fueron casi dos meses que estuve contagiada. Fue una experiencia horrible ya que las personas estaban cerca y a la vez lejos, es algo inexplicable”- Mujer, 20 años, presentó síntomas.

"Fue bien dificil porque me sentía totalmente sola, en ese momento si estaban ellos apoyándome, pero no era lo mismo. Yo pasaba llorando y no podía recibir abrazados, ni esa calidez que se siente cuando uno está acompañado.”- Mujer de 24 años, no presentó síntomas.

\section{Discusión}

El presente estudio hizo una aproximación cualitativa entorno a la experiencia del diagnóstico positivo de COVID-19. Se encontró que dicha experiencia tuvo repercusiones sobre la vida cotidiana del individuo, puntualmente en lo correspondiente al ámbito laboral-económico. En tal sentido, es posible triangular tales hallazgos con un estudio realizado en 28,850 hogares hondureños, en los que se evidencia que el $23.82 \%$ de la muestra únicamente recibía pagos parciales de su salario. Además, en dicho estudio se reporta que el 54\% de los hogares encuestados experimentó una disminución en sus ingresos económicos mensuales (Instituto de Investigaciones Económicas y Sociales, 2020). A esta situación se suman los gastos médicos provocados por el COVID-19, lo que constituye un estresor adicional para los pacientes.

En el caso de los estudiantes, el diagnóstico por COVID-19 y su respectiva incapacidad repercute en el aprovechamiento académico de los afectados, causando rezago en tal área. Esto es congruente con estudios realizados en población universitaria durante la actual pandemia, en donde 
el 86\% de los encuestados manifestaba estar preocupados por su rendimiento académico (Son, Hedge, Smith, Wang, \& Sasangohar, 2020).

Por otro lado, los informantes comentan una serie de reacciones psicológicas detonadas por el diagnóstico de COVID-19, incluyendo sentimientos de culpa. Tal hallazgo es coherente con lo expuesto en estudios previos, en donde dicha culpa se identifica como un factor de riesgo para la salud mental (Cavalera, 2020). Además de esto, los entrevistados manifiestan preocupación por la salud propia y de terceros, una reacción encontrada también en la población general (Vásquez, et al., 2020).

Los entrevistados también expresaron sentimientos de ansiedad, depresión, temor, incertidumbre y cambios cualitativos y cuantitativos en el sueño, lo que evidencia un deterioro significativo en la salud mental de los afectados. A pesar de ello, aunque algunos entrevistados admiten que hubiese sido beneficioso contar con acompañamiento psicológico profesional, la búsqueda de tal atención no fue prioritaria para los informantes. A esto se suma que los centros de triaje no cuentan con personal del campo de la psicología. Sin embargo, el discurso de los entrevistados frecuentemente posee elementos asociados a la resiliencia, puntualmente de origen espiritual. Tal hallazgo es congruente con un estudio reciente en el que se encontró una relación estadísticamente significativa entre espiritualidad y resiliencia durante la pandemia de COVID-19 (Roberto, Sellon, Cherry, Hunter-Jones, \& Winslow, 2020).

Los resultados de la presente investigación evidencian la importancia de las redes de apoyo, puntualmente en lo referente a la familia, amistades y compañeros de trabajo. En tal sentido, un estudio reciente realizado con pacientes hospitalizados por COVID-19 determinó que el apoyo de la familia, amistades y/o personal médico era un factor importante para disminuir el riesgo de depresión en dicha población. Sumado a esto, los pacientes que constantemente se comunicaban 
con otros por medio de llamadas, mensajes de texto o redes sociales, tenían un menor riesgo de presentar síntomas depresivos (Samrah, et al., 2020). Adicionalmente, cabe recalcar que en ocasiones los entrevistados reportaron sentimientos de soledad, esto debido a la necesidad de guardar distanciamiento físico y cuarentena. Tal hallazgo es de suma relevancia al considerar que la soledad se convierte en un factor de riesgo para la salud mental, en especial durante la pandemia de COVID-19 (Padmanabhanunni \& Pretorius, 2021). A esto se suma que en ocasiones los entrevistados han experimentado estigmatización debido a su diagnóstico de COVID-19. Tal situación podría exponer a los pacientes a un mayor riesgo de aislamiento, acentuando la presencia de síntomas psicológicos.

Los resultados obtenidos en esta investigación demuestran la necesidad de implementar intervenciones clínicas desde la psicología basada en evidencia, dirigidas al manejo de los síntomas que se exacerban al tener diagnóstico de COVID-19, tanto para pacientes sintomáticos como asintomáticos. Se deben crear políticas sanitarias con énfasis en un primer contacto en el espacio clínico y con seguimiento virtual; además de sistematizar la atención relacionada a duelo por COVID-19. De igual forma, se debe procurar el seguimiento de los pacientes que anteriormente ya tenían diagnósticos de salud mental para mantener el control de los trastornos y evitar recaídas. Las intervenciones para psicoeducación ante la discriminación y la estigmatización deben ser dirigidas para la población en general, por lo que los medios de comunicación juegan un papel clave para la difusión de información clínica que promueva puntos de vista alternos ante pensamientos irracionales y subjetivos.

A pesar de los aportes de la presente investigación, hay limitaciones que deben ser consideradas. En primer lugar, la naturaleza no probabilística de la selección de informantes, así como el método utilizado, no permiten la generalización de resultados a la población. En segunda 
instancia, por motivos de bioseguridad, el levantamiento de información se llevó a cabo por medios virtuales, de manera que personas sin acceso a internet no pudieron ser parte del presente estudio, limitando la pluralidad de la muestra. Futuras investigaciones se beneficiarían de la utilización de métodos mixtos, en donde se realicen abordajes cuantitativos con pruebas de tamizaje, además de entrevistas a profundidad con personas con diagnóstico positivo de COVID-19. Adicionalmente, considerando el riesgo físico y psicológico en el que se encuentra el personal de salud, próximas investigaciones deberán estudiar a profundidad los efectos del diagnóstico positivo en tal personal, además de los efectos del daño moral asociado al ejercicio profesional.

\section{Declaración de conflicto de interés}

Los autores declaran no tener conflictos de interés.

\section{Fuentes de financiamiento}

Ninguna.

\section{Acerca de la autoría}

Miguel Landa-Blanco: Conceptualización, análisis formal, investigación, metodología, administración del proyecto, supervisión, validación, redacción-borrador original.

Elizabeth Andino-Rodríguez, Gabriela Vásquez y Miguel Ángel Ruch-Ordoñez: Conceptualización, análisis formal, investigación, metodología, validación, redacción-borrador original. 
Reacciones psicológicas ante el diagnóstico de COVID-19: un estudio cualitativo

\section{Referencias}

Cavalera, C. (2020). COVID-19 Psychological Implications: The Role of Shame and Guilt. Frontiers in Psychology, 11. doi:10.3389/fpsyg.2020.571828

Centros para el Control y la Prevención de Enfermedades [CDC]. (2020, Noviembre 30). CDC. Retrieved from Centros para el Control y la Prevención de Enfermedade: https://espanol.cdc.gov/coronavirus/2019-ncov/need-extraprecautions/index.html?CDC_AA_refVal=https\%3A\%2F\%2Fwww.cdc.gov\%2Fcoronavi rus\%2F2019-ncov\%2Fneed-extra-precautions\%2Fpeople-at-increased-risk.html

Hernández, R., Fernández, C., \& Baptista, M. P. (2014). Metodología de la Investigación (Sixth ed.). México D.F., México: McGraw-Hill Education.

Hui, D. S., \& Zumla, A. (2019). Severe Acute Respiratory Syndrome: Historical, Epidemiologic, and Clinical Features. Infectious Disease Clinics: Emerging and Re-Emerging Infectious Diseases, 869-889.

Instituto de Investigaciones Económicas y Sociales. (2020). COVID-19 en Honduras: Análisis de Percepción de Impacto. Tegucigalpa: UNAH. Retrieved from https://iies.unah.edu.hn/assets/Uploads/PIEF-Hogares-Universitarios-COVID19Nacional3.pdf

Majadas, S., Pérez, J., Casado-Espada, N., Zambrana, A., Bullón, A., \& Roncero, C. (2020). Case with psychotic disorder as a clinical presentation of COVID-19. Psychiatry and Clinical Neurosciences, 74(10), 551-552. doi:10.1111/pcn.13107 
Reacciones psicológicas ante el diagnóstico de COVID-19: un estudio cualitativo

McBride, C., Clipp, E., Peterson, B., Lipkus, I., \& Demark-Wahnefried, W. (2000). Psychological impact of diagnosis and risk reduction among cancer survivors. Psychooncology, 9(5), 418427. doi:10.1002/1099-1611(200009/10)9:5<418::aid-pon474>3.0.co;2-e

Muhidin, S., Vizheh, M., \& Moghadam, Z. (2020). Anticipating COVID-19 related stigma in survivors and health-care workers: Lessons from previous infectious diseases outbreaks An integrative literature review. Psychiatry and Clinical Neurosciences, 74(11), 617-618. doi:10.1111/pen.13140

OMS. (2020, Junio 29). Organización Mundial de la Salud. Retrieved from Listings of WHO's response to COVID-19: https://www.who.int/news/item/29-06-2020-covidtimeline

OMS. (2020). Weekly epidemiological update - 29 December 2020. doi:https://www.who.int/publications/m/item/weekly-epidemiological-update---29december-2020

Organización Mundial de la Salud/Organización Panamericana de la Salud (OMS/OPS). (2020, enero 29). Requerimientos para uso de equipos de Equipo Protección Personal (EPP) para el nuevo coronavirus (2019 n-COV) en establecimientos de salud. Retrieved from Organización Mundial de la Salud/Organización Panamericana de la Salud (OMS/OPS): https://www.paho.org/es/documentos/requerimientos-para-uso-equipos-proteccionpersonalepp-para-nuevo-coronavirus-2019-ncov

Padmanabhanunni, A., \& Pretorius, T. (2021). The unbearable loneliness of COVID-19: COVID19-related correlates of loneliness in South Africa in young adults. Psychiatry Research, 296. doi:10.1016/j.psychres.2020.113658 
Reacciones psicológicas ante el diagnóstico de COVID-19: un estudio cualitativo

Paz, C., Mascialino, G., Adana-Díaz, L., Rodríguez-Lorenzana, A., Simbaña-Rivera, K., GómezBarreno, L., ... Ortiz-Prado, E. (2020). Anxiety and depression in patients with confirmed and suspected COVID-19 in Ecuador. Psychiatry and Clinical Neurosciences, 47(10), 554555. doi:10.1111/pcn.13106

Roberto, A., Sellon, A., Cherry, S., Hunter-Jones, J., \& Winslow, H. (2020). Impact of spirituality on resilience and coping during the COVID-19 crisis: A mixed-method approach investigating the impact on women. Health Care for Women International, 41(11-12), 1313-1334. doi:10.1080/07399332.2020.1832097

Sahoo, S., Mehra, A., Dua, D., Suri, V., Malhotra, P., Yaddanapudi, L., . . Grover, S. (2020). Psychological experience of patients admitted with SARS-CoV-2 infection. Asian Journal of Psychiatry, 54. doi:10.1016/j.ajp.2020.102355

Samrah, S., Al-Mistarehi, A., Aleshawi, A., Khasawneh, A., Momany, S., Momany, B., . . . Khassawneh, B. (2020). Depression and Coping Among COVID-19-Infected Individuals After 10 Days of Mandatory in-Hospital Quarantine, Irbid, Jordan. Psychology Research and Behavior Management, 13, 823-830. doi:10.2147/PRBM.S267459

Secretaría de Salud de Honduras [SESAL]. (2020, Octubre 21). Secretaría de Salud de Honduras [SESAL]. Retrieved from Protocolo de Manejo Clinico de Paciente Adulto con COVID-19 Segun Etapas de la Enfermedad REV 2 Septiembre 2020: http://www.salud.gob.hn/site/index.php/component/edocman/sesal-protocolo-de-manejoclinico-de-paciente-adulto-con-covid-19-segun-etapas-de-la-enfermedad-rev-2septiembre-2020 
Reacciones psicológicas ante el diagnóstico de COVID-19: un estudio cualitativo

Secretaria de Salud de Honduras. (2020, marzo 23). Lineamientos generales para el uso del Equipo de Protección Personal (EPP), para prevenir la exposición a Coronavirus (COVID-19)y su transmisión durante las actividades de notificaciones de actos administrativos de.

Retrieved

from http://www.essalud.gob.pe/ietsi/pdfs/guias/Recomendaciones_para_el_uso_de_EPP_CO VID_19.pdf

Secretaria de Salud de Honduras. (2020b, Marzo 10). COVID-19 Honduras. Retrieved from Salud confirma los dos primeros casos de COVID-19 en el territorio hondureño: https://covid19honduras.org/?q=pdf-primeros-dos-casos

Shereen, M. A., Khan, S., Kazmi, A., Bashir, N., \& Siddique, R. (2020). COVID-19 infection: Origin, transmission, and characteristics of human coronaviruses. Journal of Advanced Research, 24, 91-98. doi:10.1016/j.jare.2020.03.005

Son, C., Hedge, S., Smith, A., Wang, X., \& Sasangohar, F. (2020). Effects of COVID-19 on College Students' Mental Health in the United States: Interview Survey Study. Journal of Medical Internet Research, 22(9). doi:10.2196/21279

Unidad de Vigilancia de la Salud [UVS]. (2021). Informe Epidemiológico- Pandemia por SARSCoV-2 (COVID-19). Tegucigalpa, Distrito Central: Secretaría de Salud de Honduras [SESAL].

Urzúa, A., Vera-Villarroel, P., Caqueo-Urízar, A., \& Polanco-Carrasco, R. (2020). La Psicología en la prevención y menejo del COVID-19. Aportes desde la evidencia inicial. Terapia Psicológica, 38(1), 103-118. Retrieved from scielo.conicyt.cl/pdf/terpsicol/v38n1/07184808-terpsicol-38-01-0103.pdf 
Reacciones psicológicas ante el diagnóstico de COVID-19: un estudio cualitativo

Vásquez, G., Urtecho-Osorto, Ó. R., Agüero-Flores, M., Díaz Martínez, M. J., Paguada, R. M., Varela, M., . . Echenique, Y. (2020). Mental health, confinement, and coronavirus concerns: a qualitative study. . Revista Interamericana De Psicología/Interamerican Journal of Psychology, 54(2). doi:10.30849/ripijp.v54i2.1333

Zandifar, A., \& Badrfam, R. (2020). Exacerbation of psychosis accompanied by seizure and catatonia in a patient with COVID-19: A case report. Psychiatry and Clinical Neurosciences. doi:10.1111/pcn.13174

Zhang, J., Lu, H., Zeng, H., Zhang, S., Du, Q., Jiang, T., \& Du, B. (2020). The differential psychological distress of populations affected by the COVID-19 pandemic. Brain, Behavior, and Immunity, 87, 49-50. doi:10.1016/j.bbi.2020.04.031 